\title{
EasyCatch, a convenient, sensitive and specific CRISPR detection system for cancer gene mutations
}

\author{
Yin Liu', Yanling Chen', Lu Dang², Yixin Liư3, Shisheng Huang ${ }^{4}$, Sanyun Wu', Peixiang Ma ${ }^{5}$, Hongqiang Jiang ${ }^{1}$, \\ Yi Li ${ }^{3}$, Yunbao Pan ${ }^{6}$, Yongchang Wei ${ }^{3}$, Xiaodong Ma ${ }^{7}$, Ming Liü ${ }^{8}$, Quanjiang $\mathrm{Ji}^{9}$, Tian Chi ${ }^{4}$, Xingxu Huang ${ }^{4 *}$, \\ Xinjie Wang ${ }^{10^{*}}$ and Fuling Zhou ${ }^{1 *}$
}

Keywords: CRISPR detection, cancer mutation, Drug resistance, Leukemia, SNP detection

\section{Main text}

Convenient, sensitive and specific detection of rare genetic variants and mutations is essential for early cancer diagnosis and precision medicine [1], but tools that are simultaneously endowed with all these attributes remain elusive despite years of intense quest. Recently, Clustered Regularly Interspaced Short Palindromic Repeats (CRISPR) associated (Cas) proteins have shown great potential for rapid and sensitive nucleic acid detection [2-5]. Guided by a CRISPR-derived RNA (crRNA) complementary to the target sequence, the CRISPR/Cas complex can recognize and cleave the target nucleic acid with single-base resolution specificity [6]. Cas12a is one of the most commonly used CRISPR/Cas proteins for DNA detection. Upon recognition and cleavage of target double-strand DNA, the collateral cleavage activity of Cas12a is activated, resulting in cleavage of nearby single-strand DNA (including fluorescence reporters) in a

\footnotetext{
*Correspondence: huangxx@shanghaitech.edu.cn; wang_xin_jie@126.com; zhoufuling@whu.edu.cn

${ }^{1}$ Department of Hematology, Zhongnan Hospital of Wuhan University, No.169 Donghu Road, Wuhan 430072, China

${ }^{4}$ Gene Editing Center, School of Life Science and Technology,

ShanghaiTech University, 100 Haike Rd., Pudong New Area,

Shanghai 201210, China

${ }^{10}$ Shenzhen Branch, Guangdong Laboratory of Lingnan Modern Agriculture, Genome Analysis Laboratory of the Ministry of Agriculture and Rural Affairs, Agricultural Genomics Institute at Shenzhen, Chinese Academy of Agricultural Sciences, Shenzhen, China

Full list of author information is available at the end of the article
}

non-specific manner [7], and this "collateral cleavage" has been widely exploited for sensitive and specific detection of target sequences $[8,9]$. However, for detection of rare mutations in cancers, the majority of the DNA are wildtype (WT) sequences, significantly hampering the analysis. We have now solved the problem by simply including restriction digestion in the detection system, making our method (EasyCatch, for Excision-amplification-synchronous Cas12a-targeted checkout) the first capable of convenient, specific, sensitive $(0.001 \%)$, and rapid $(<1 \mathrm{~h})$ detection of mutations in cancer samples.

\section{Development of EasyCatch}

For traditional CRISPR detection, when the target mutation sequence in the samples constitutes only a minority of total DNA, the detection becomes difficult due to the interference by the non-target WT sequences, even after target enrichment by recombinase polymerase amplification (RPA, in $20 \mathrm{~min}$ at $37 \sim 42^{\circ} \mathrm{C}$ ) (Fig. 1a) [10]. We hypothesized that by adding a restriction enzyme recognizing the WT sequence to the RPA system, we can destroy the interfering sequences, and thereby inhibit the amplification of the WT sequences while facilitate the amplification of mutant templates, thus increasing the detection sensitivity (Fig. 1a). This strategy is the basis of EasyCatch.

For proof-of-concept demonstration, we used EasyCatch to detect the drug-resistant FLT3 gene D835Y/H/ $\mathrm{V} / \mathrm{F}$ mutations in acute myeloid leukemia (AML) [11]. original author(s) and the source, provide a link to the Creative Commons licence, and indicate if changes were made. The images or other third party material in this article are included in the article's Creative Commons licence, unless indicated otherwise in a credit line to the material. If material is not included in the article's Creative Commons licence and your intended use is not permitted by statutory regulation or exceeds the permitted use, you will need to obtain permission directly from the copyright holder. To view a copy of this licence, visit http://creativecommons.org/licenses/by/4.0/. The Creative Commons Public Domain Dedication waiver (http://creativeco mmons.org/publicdomain/zero/1.0/) applies to the data made available in this article, unless otherwise stated in a credit line to the data. 
Since EcoRV restriction enzyme can recognize and digest WT D835 sequence (-GATATC-), but not the D835 mutant sequences (Fig. S1a). The feasibility of EcoRV digestion in the RPA reaction was validated using PCR fragments, wherein the fluorescence signal of WT D835 fragments was eliminated by EcoRV digestion, while that of the $\mathrm{D} 835 \mathrm{Y} / \mathrm{H} / \mathrm{V} / \mathrm{F}$ fragments was enhanced (Fig. S1b-d).

In a Cas12a reaction, a mutation-specific crRNA is required to avoid the cross-reactivity against WT. To screen for the optimal crRNA for detecting FLT3D835Y, we designed four crRNAs, with FLT3-D835YcrRNA1 perfectly matching the mutant sequence and FLT3-D835Y-crRNA2 4 bearing various mismatches (Fig. 1b). We compared the sensitivity and specificity of these four crRNAs in detecting D835Y in PCR fragments (1e10 copies) comprising the D835Y and WT alleles. After $30 \mathrm{~min}$ of the Cas12a reaction, all the 4 crRNAs, particularly crRNA 1 and crRNA2, could detect the sample with $100 \%$ D835Y, while crRNA1 but not crRNA2 produced a strong signal even for WT sample (Fig. 1c; S2). Thereby crRNA2 is the optimal crRNA for D835Y detection based on its excellent sensitivity and specificity. Optimal crRNAs for D835H/V/F and WT were similarly identified, which are D835H-crRNA2, D835V-crRNA2, D835F-crRNA, WT-crRNA2, respectively (Fig. 1d; S3-6), and an inner control (IC)-crRNA was also designed to target a non-mutation sequence near the mutation site. To simplify the diagnosis of the four mutations, we pooled the $\mathrm{D} 835 \mathrm{Y} / \mathrm{H} / \mathrm{V} / \mathrm{F}$ crRNAs (MMT-crRNAs) and the 4 mutant fragments into a single reaction, resulting in a strong fluorescence signal, whereas the WT sample did not show any signal, as expected (Fig. 1e, f; S7).

Next, we screened 9 pairs of RPA primers to improve EasyCatch sensitivity (Fig. S8). Using the best primers and MMT-crRNAs, as low as 10 copies of D835Y plasmid templates could be detected only after $20 \mathrm{~min}$ of RPA and $20 \mathrm{~min}$ of Cas12a reaction (Fig. 1g). Therefore, we chose this primer pair and 20min as the Cas12a reaction time in the subsequent experiments.

\section{EasyCatch achieves a $0.001 \%$ sensitivity in FLT3-D835Y detection}

With the optimized crRNAs and primers, we set out to determine the detection limit of EasyCatch for FLT3-D835Y. We first quantified the effect of EcoRV on WT sequence, finding that EcoRV could completely inhibit the amplification of up to $1 \mathrm{e} 6$ copies of WT templates but spared the mutant target as expected (Fig. S9, 10). We then mixed the mutant and WT templates at various ratios, with FLT3D835Y comprised 100, 50, 25, 10, 1, 0.1, 0.01, and 0.001\% of the total templates, and used 1e6 copies of the templates as the input for EasyCatch, finding the detection limit being $0.001 \%$ (i.e., 10 copies of D835Y template amid 999,990 copies of WT template). In contrast, in the absence of EcoRV, namely CRISPR detection, the detection limit was 1000x lower (1\%) (Fig. 1h, i; S11). Finally, FGS directly demonstrated that EcoRV markedly enriched the mutant allele in the RPA mixture from 10,1 and $0.1 \%$ to 100,98 and $51 \%$, respectively (Fig. 1j).

We next compared EasyCatch with the commonly used qPCR-based detection method. We designed and tested two D835Y-specific TaqMan probes (Fig. S12), and then chose the more specific one (probe 1) for the comparison (Fig. S13, 14). The qPCR results showed that the amplification curves and Ct values of $1,0.1,0.01 \%$ mutant samples cannot be distinguished from that of the WT sample (Fig. $1 \mathrm{k}, \mathrm{l}$ ), indicating its sensitivity of only $1 \% \sim 10 \%$. Together, these results suggested that EasyCatch is much more sensitive than TaqMan qPCR in detecting FLT3D835Y mutation.

\section{EasyCatch accurately detected FLT3-D835Y/V/H/F mutations in clinical samples}

With these, AML patient samples were used for EasyCatch detection of D835Y/V/H/F mutations. Briefly, $200 \sim 500 \mu \mathrm{l}$ of blood was incubated with red blood cell (RBC) lysis buffer for $1 \mathrm{~min}$, and the cell precipitate was obtained by 1 min of centrifugation. Next, genomic DNA was released by a nucleic acid releaser under $95^{\circ} \mathrm{C}$ for $3 \mathrm{~min}$ and then treated by the EasyCatch assay (Fig. S15). From drawing blood to making treatment

\footnotetext{
(See figure on next page.)

Fig. 1 Development and validation of EasyCatch system for specific and sensitive mutation detection. a Schemes of EasyCatch compared with CRISPR detection. $\mathbf{b}$ Sequences of D835Y-crRNAs, and FLT3-WT and D835Y gene region. Base G mutates to T in D835Y. c Fluorescence heatmap of different D835Y-crRNA-induced Cas12a reactions detecting 1e10 copies of PCR fragments with different D835Y mutation rates (100, 50, 10\%, and 0), Cas12a reactions for 10, 20 and 30 min were recorded. $\mathbf{d}$ Specificity assay of D835Y-crRNA2, D835H-crRNA, D835V-crRNA, and D835F-crRNA. Time-course of fluorescence intensity and naked-eye observation after $60 \mathrm{~min}$ of Cas 12a reaction are shown. e Schematic diagram of MMT-crRNAs-guided Cas12a reaction to identify D835Y/H/N/F mutations from WT background. f Specificity assay of MMT-crRNAs using 1 e10 copies of D835Y/H/N/F and WT fragments. Fluorescence intensity after $60 \mathrm{~min}$ of Cas 12a reaction are shown. IC, inner control. $\mathbf{g}$ Time-course analysis of the detection of 1e1 D835Y plasmid templates by RPA combined with MMT-crRNAs induced Cas 12a reaction. $\mathbf{h}$, i Sensitivity comparison of EasyCatch and CRISPR detection in detecting 1 e6 copies of plasmid templates with gradient D835Y mutation rates. $\mathbf{j}$ FGS results of the amplified products in EasyCatch and CRISPR detection. The D835Y mutation rates were quantified using the online tool EditR (https://moriaritylab.shinyapps.io/editr_ v10/). k The design and amplification plot of D835Y-probe-mediated qPCR in detecting 1 e6 copies of plasmid templates with gradient D835Y mutation rates. I Ct value comparison of different qPCR samples
} 

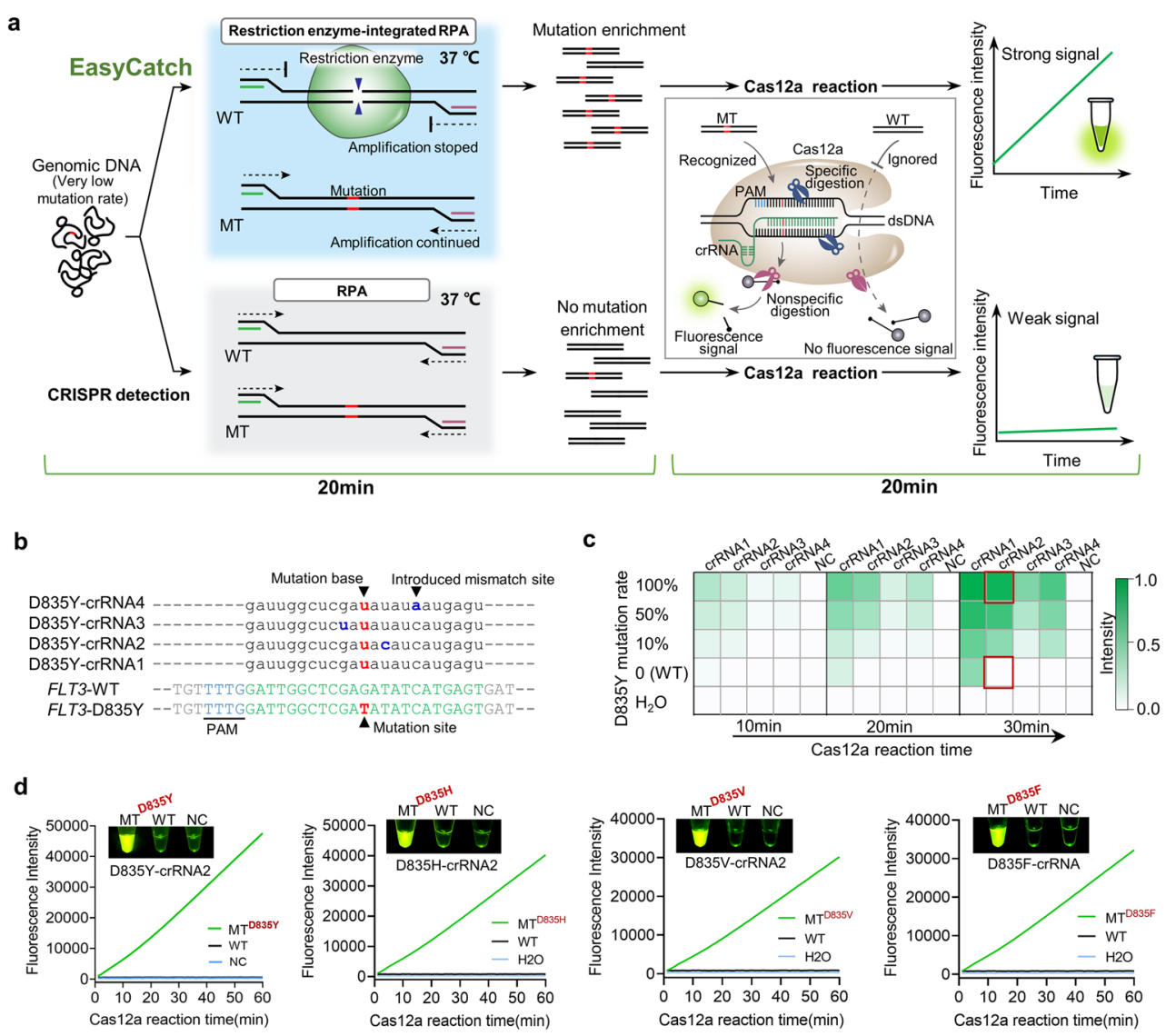
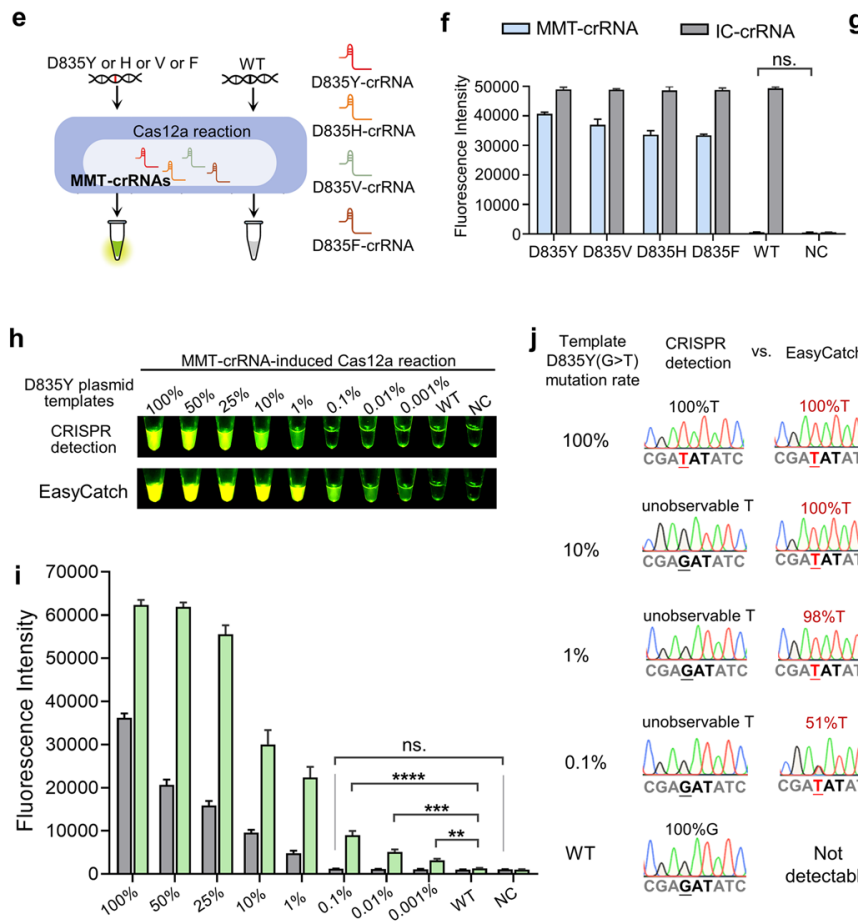

Fig. 1 (See legend on previous page.)
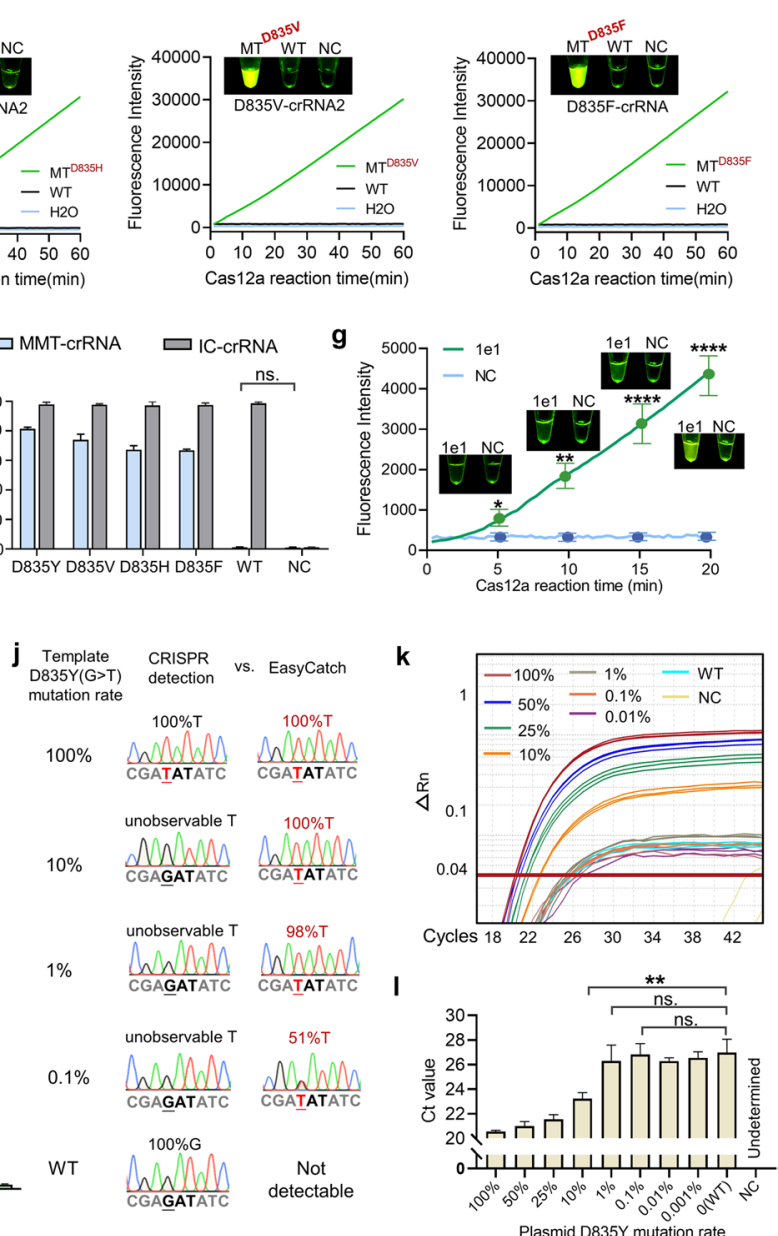
decisions, the whole process can be completed within $1 \mathrm{~h}$ (Fig. 2a). We then simultaneously applied EasyCatch and first-generation sequencing (FGS) to detect $32 \mathrm{AML}$ samples whose FLT3 gene mutation status had been analyzed by next-generation sequencing (NGS) previously, wherein P6, P12, P17, P27, and P31 carried drugresistant D835Y/V/H/F mutations (Fig. 2b; S16, 17). The results showed that EasyCatch successfully identified all five mutant samples; in contrast, only two samples with relatively high mutation rates, P12 of $17.2 \%$ and P31 of $10.9 \%$, were identified by FGS (Fig. 2b). Thus, EasyCatch outperforms FGS for clinical sample analysis. More importantly, the EasyCatch method is simple and economical, and only needs a mini centrifuge, a $20 \mu \mathrm{l}$ pipette and tips, a heat blocker, and a blue lamp with a $485 \mathrm{~nm}$ wavelength (Fig. S18).

We next benchmarked EasyCatch against the commonly used FGS for detecting FLT3-D835 mutations in 80 AML patients (P33 -P112) with unknown FLT3-D835 mutation status, with NGS as the gold standard. EasyCatch, but not FGS, was able to detect D835Y in P38, P59, P71, P80, P83, and P106 (Fig. 2c). NGS confirmed that all the six samples harbored the D835Y mutation (at 4.5, 2.7, 4.1, 3.5, 1.2 and 2.4\%, respectively) (Fig. S19, 20). Notably, NGS showed that P86 carried $10.9 \%$ nondrug-resistant D835E (GAT>GAA) mutation. As this mutation did not produce a signal in EasyCatch, further confirming the high specificity of this method (Fig. 2c). The statistical analysis of the 80 cases further showed that EasyCatch is much more sensitive than FGS (100\% vs. 0\%) (Fig. 2d).

\section{EasyCatch is applicable to other cancer mutations}

To verify the versatility of EasyCatch, we applied it to detect 4 mutations at 3 other genes (IDH2, EGFR and NRAS). IDH2 R172K is a hotspot mutation in glioma and leukemia, and of prognostic and therapeutic value $[12,13]$. EGFR e19del and L858R are the two main mutations sensitive to EGFR-TKIs, thus of great therapeutic value for patients with lung cancer [14]. At the same time, the NRAS G12D is a driver mutation in leukemia and colorectal cancer [15]. All four mutations are important testing items in the clinic. We first compared EasyCatch with the CRISPR detection method to detect 1 e 5 copies of plasmid templates of 1 and $0.1 \%$ mutation rate. The results showed that the WT fluorescence signals were strong while the signals of all the four mutations were weak in CRISPR detection. However, the WT signals were almost invisible while the mutation signals were significantly increased in EasyCatch (Fig. 2e; S21a). The FGS results of the amplified products also confirmed the excellent mutation enrichment effect of EasyCatch (Fig. S21b). Further analysis indicated that the MT/WT fluorescence ratios in EasyCatch were hundreds of times higher than that of CRISPR detection (Fig. 2f). More samples with decreasing frequencies $(100,50,25,10,1,0.1 \%$, and 0$)$ of these mutations were also tested, with consistent results (Fig. S22-25).

We also detected EGFR-e19del, EGFR-L858R, and NRAS-G12D mutations using commercial kits based on fluorescence qPCR. The tested samples were 1e5 copies of plasmid templates with a mutation rate of $10,1,0.1$, and $0 \%$ (WT), respectively. The results of all three sites showed that the amplification curves of different samples were gradually shifted to right, consistent with the decreased mutation rate. However, we noticed strong fluorescence signals in WT samples (Fig. 2g-i; S26-28). In our EasyCatch detection, the WT signal was completely inhibited by restriction digestion and mutation-specific crRNA, ensuring the reliability of the results.

Finally, we predicted the scope of application of EasyCatch. Total 91,771 human disease-related sites have been documented in the ClinVar database (www.ncbi. nlm.nih.gov/clinvar), and more than 63,000 restriction enzymes reported in the REBASE database (http:// rebase.neb.com/rebase/azlist.re2.html), 485 of them commercially available and functioning at $37^{\circ} \mathrm{C}$. We

\footnotetext{
(See figure on next page.)

Fig. 2 EasyCatch is applicable to clinical samples and other cancer mutations. a Schematic diagram of the whole mutation diagnosis. b EasyCatch and FGS results of 32 AML samples with known FLT3-D835 mutation status, 10/32 cases are shown. D835YN/H/F-positive patients are marked by red IDs, and red triangles indicate mutant bases. c EasyCatch, FGS, and NGS results of 80 AML samples with unknown FLT3-D835 mutation status, $9 / 80$ cases are shown. WT and mutated bases in NGS are marked by green and red, respectively. $\mathbf{d}$ Statistical table of the sensitivity and specificity of EasyCatch compared with FGS using NGS as a standard reference. e Sensitivity comparison between EasyCatch and CRISPR detection in the detection of IDH2-R172K, EGFR-e19del and L858R, and NRAS-G12D mutations. Genomic locations of these mutations were shown above, wherein exons and mutation sites were colored in blue and red, respectively. The tested samples were 1 e 5 copies of plasmid templates with a mutation rate of $0.1 \%$. Each amplified product was detected by both WT-crRNA and mutation-crRNA induced Cas 12a reaction. Fluorescence intensity and naked eye results were both recorded. $\mathbf{f}$ Statistic analysis of the MT/WT fluorescence ratio in EasyCatch and CRISPR detection. The results of 1 and $0.1 \%$ mutated samples were counted together. $\mathbf{g}, \mathbf{h}$, i The qPCR assay for EGFR-e19del, L858R and NRAS-G12D detection, respectively. The qPCRs were performed on 10, 1 and $0.1 \%$ mutated templates. A $100 \%$ WT template and $\mathrm{dd}_{2} \mathrm{O}(\mathrm{NC})$ served as control. $\mathbf{j}$ The statistical chart of restriction enzyme cuttable human disease-related genomic sites (mutation $<27 \mathrm{bp}$, which is the detection length of crRNA), wherein commercial available $37^{\circ} \mathrm{C}$ restriction enzyme cuttable sites can be potentially detected by EasyCatch. The inclusion relation is shown in the upper-right corner
} 
a

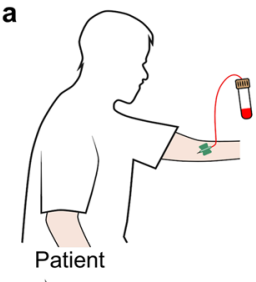

Suitable treatment O

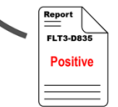

200 500 $\mu \mathrm{l}$

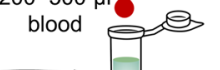

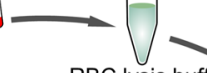
$\underset{\substack{\text { R min } \\ \text { MBC lysfer }}}{\theta}$ $\square<1 h$ $2<<1 \mathrm{~h}$

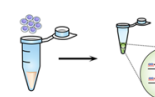
$\frac{\text { EasyCatch }}{45 \mathrm{~min}} \frac{\pi}{\nabla \theta+1}$ b
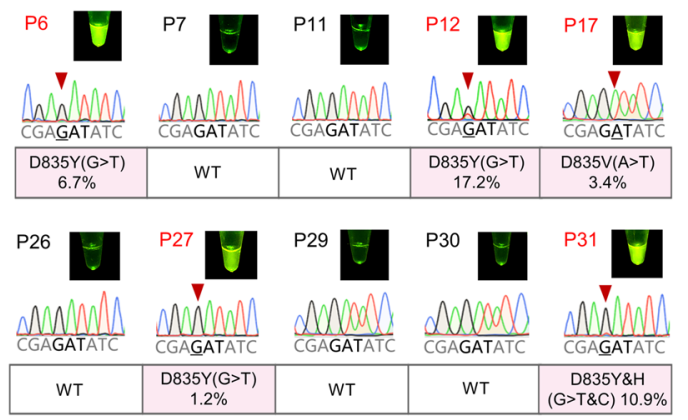

c

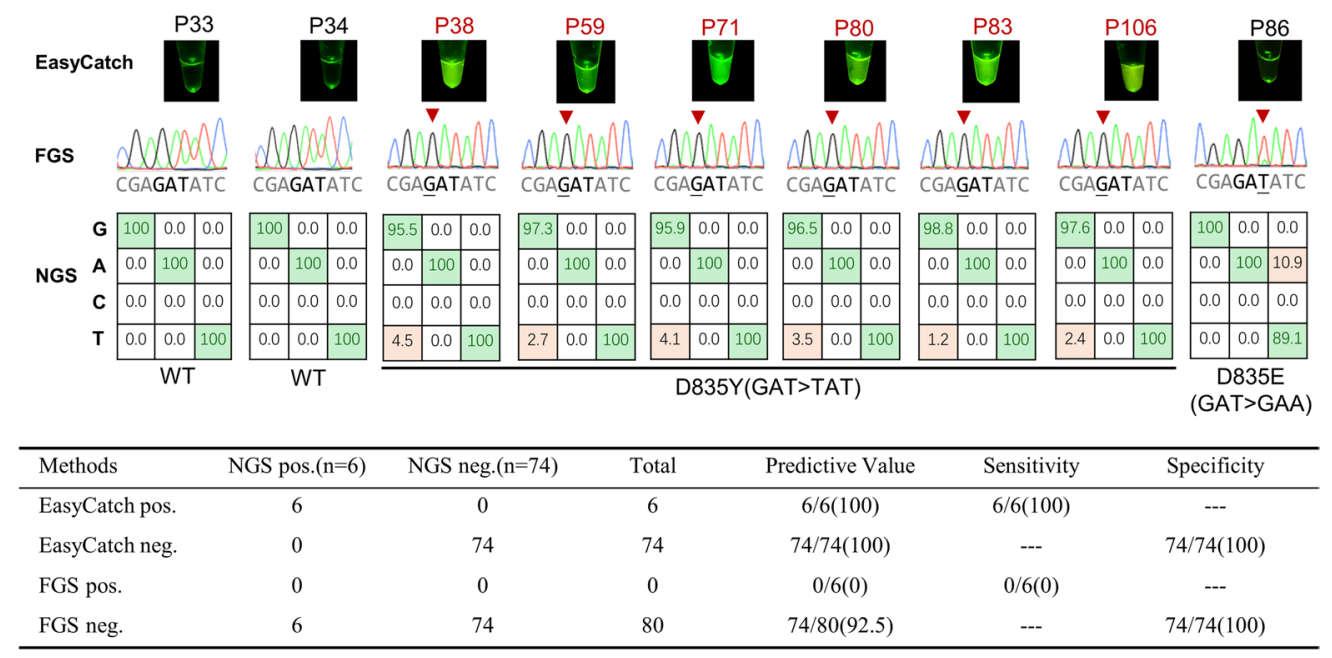

e

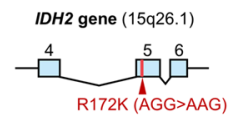

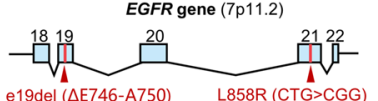

NRAS gene (1p13.2) e19del (AE746-A750) L858R (CTG>CGG)
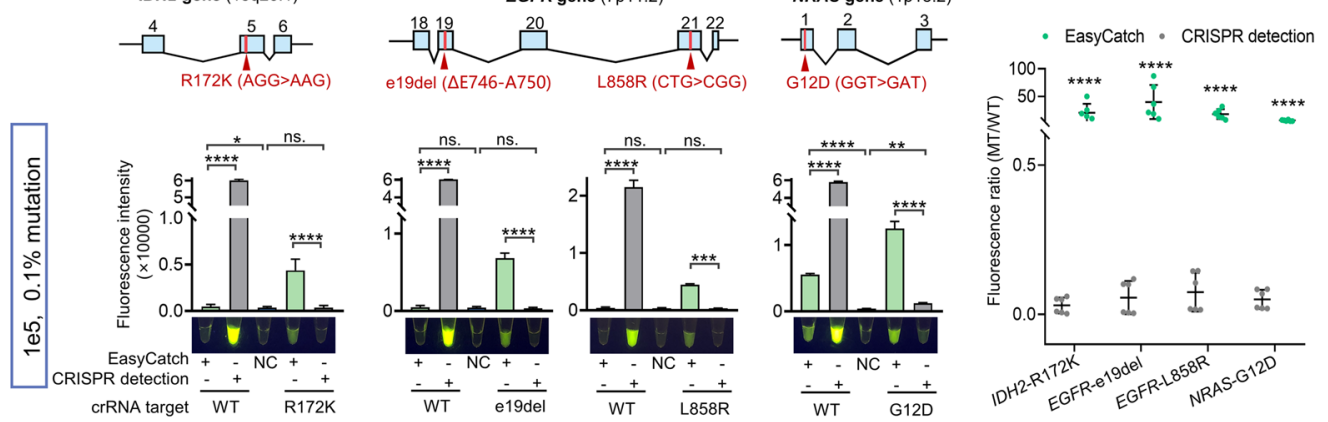

g
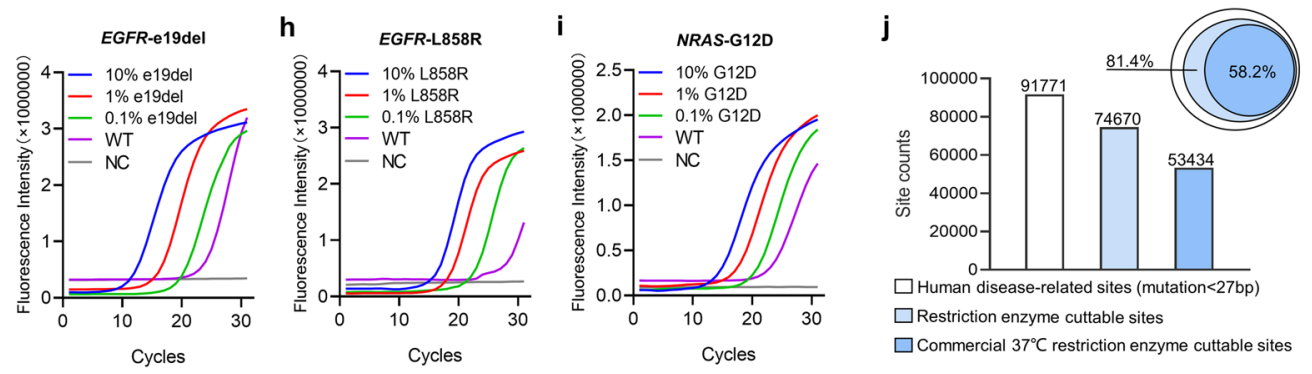

Fig. 2 (See legend on previous page.) 
found that 53,434 (58.2\%) sites can be cut by the 485 enzymes, indicating that the EasyCatch system can potentially diagnose $\sim 60 \%$ of human disease-related mutations (Fig. 2j). In addition to restriction enzymes, PAM requirement also imposes restriction on the target range of EasyCatch, but this can be alleviated using Cas12a variants (Table S5), and primer-introduced PAM (Fig. S29). Thus, EasyCatch is a versatile method for detecting gene mutations.

\section{Conclusions}

We have adapted the Cas12a-based DNA detection platform for evaluating rare cancer-related mutations by establishing EasyCatch assay with the sensitivity of $0.001 \%$, which can be completed (from sample preparation to data output) within an hour using only simple instruments and operations. Our study established EasyCatch as the first mutation detection method that is not only superbly sensitive and specific, but also extremely fast, simple, and convenient, showing its versatility in point-of-care cancer diagnosis and precision medicine.

\section{Abbreviations \\ CRISPR: Clustered Regularly Interspaced Short Palindromic Repeats; Cas: CRISPR associated proteins; AML: Acute myeloid leukemia; FLT3: FMS-like tyros- ine kinase 3; PCR: Polymerase chain reaction; WT: Wild-type; crRNA: CRISPR RNA; RPA: Recombinase polymerase amplification; RBC: Red blood cell; WBC: White blood cell; FGS: First-generation sequencing; NGS: Next-generation sequencing.}

\section{Supplementary Information}

The online version contains supplementary material available at https://doi. org/10.1186/s12943-021-01456-x

\section{Additional file 1. Methods.}

Additional file 2: Supplementary Fig. 1 Validation of the activity and specificity of EcoRV in RPA system. Supplementary Fig. 2 Specificity assay of FLT3-D835Y-crRNAs. Supplementary Fig. 3 Specificity assay of FLT3-D835H-crRNAs. Supplementary Fig. 4 Specificity assay of FLT3-D835V-crRNAs. Supplementary Fig. $\mathbf{5}$ Specificity assay of FLT3-D835F-crRNAs. Supplementary Fig. 6 Specificity assay of FLT3D835WT-crRNAs. Supplementary Fig. 7 Specificity assay of MMT-crRNAs. Supplementary Fig. 8 RPA primer screen for highly sensitive EasyCatch detection. Supplementary Fig. $\mathbf{9}$ WT inhibition assay by RPA with or without EcoRV. Supplementary Fig. 10 Detection of 1e6 1e1 copies of D835Y and WT plasmids using RPA with or without EcoRV. Supplementary Fig. 11 Inhibition of WT amplification by EasyCatch. Supplementary Fig. 12 Design of TaqMan qPCR for the detection of FLT3-D835Y. Supplementary Fig. 13 The amplification plot of D835Y-probe 1-involved qPCR in detecting 1e5 copies of plasmid templates with gradient D835Y mutation rates. Supplementary Fig. 14 The amplification plot of D835Yprobe 2-involved qPCR in detecting 1 e5 copies of plasmid templates with gradient D835Y mutation rates.

Additional file 3: Supplementary Fig. 15 Exploration of the fastest blood processing time before EasyCatch. Supplementary Fig. 16 Classification information and NGS results of the FLT3-D835 mutation status of 32 AML samples. Supplementary Fig. 17 EasyCatch and FGS results of the $32 \mathrm{AML}$ samples in the detection of FLT3-D835YN/H/F nutations. Supplementary Fig. 18 Equipment needed in EasyCatch assay.
Supplementary Fig. 19 EasyCatch results of 80 AML patient samples read by naked eyes. Supplementary Fig. 20 EasyCatch, FGS and NGS results of 80 AML patient blood samples. Supplementary Fig. 21 Broad application of EasyCatch in cancer mutation diagnosis. Supplementary Fig. 22 Comparison of CRISPR detection and EasyCatch on IDH2-R172K mutation. Supplementary Fig. 23 Comparison of CRISPR detection and EasyCatch on EGFR-e19del mutation. Supplementary Fig. 24 Comparison of CRISPR detection and EasyCatch on EGFR-L858R mutation. Supplementary Fig. 25 Comparison of CRISPR detection and EasyCatch on NRAS-G12D mutation. Supplementary Fig. 26 The amplification plot of fluorescence qPCR detection of EGFR gene e19del mutation using a commercial kit. Supplementary Fig. 27 The amplification plot of fluorescence qPCR detection of EGFR gene L858R mutation using a commercial kit. Supplementary Fig. 28 The amplification plot of fluorescence qPCR detection of EGFR gene L858R mutation using a commercial kit. Supplementary Fig. 29 The RPA primer design for IDH2-R172K mutation detection.

Additional file 4: Supplementary Table 1 PCR and RPA primer sequences. Supplementary Table 2 crRNA sequences. Supplementary Table 3 Next-generation sequencing primer sequences. Supplementary Table 4 Primers and probes of Taqman qPCR. Supplementary Table 5

Cas12a nuclease natural and engineered variants.

\section{Acknowledgements}

We thank members of Huang lab and Zhou lab for helpful discussions. We also thank the Molecular and Cell Biology Core Facility (MCBCF) and the Molecular Imaging Core Facility (MICF) at the School of Life Science and Technology, ShanghaiTech University for providing technical support.

\section{Authors' contributions}

Y.L., F. Z., X.W., and X.H. conceptualized the study; Y.L. performed the main research and wrote the original draft with the assistance of other authors. F.Z., X.W., and X.H. supervised the project, and revised the manuscript. The author(s) read and approved the final manuscript.

\section{Funding}

This study was supported by the National Natural Science Foundation of China (Grant No. 81770179, 81830004 and 82002144), public welfare scientific research project of Zhongshan City (2019B1015), clinical technological innovation project of Wuhan University (Icyf202010), and emergency key program of Guangzhou Laboratory (EKPG21-18).

\section{Availability of data and materials}

The data supporting the conclusions of this article have been given in this article and its additional files, and can also be requested from the corresponding authors.

\section{Declarations}

Ethics approval and consent to participate

This study was approved by Zhongnan Hospital of Wuhan University, and School of Life Science and Technology, ShanghaiTech University.

\section{Consent for publication}

All authors have agreed to publish this manuscript.

\section{Competing interests}

The authors declare that they have no competing interests.

\section{Author details}

${ }^{1}$ Department of Hematology, Zhongnan Hospital of Wuhan University, No.169 Donghu Road, Wuhan 430072, China. ${ }^{2}$ Department of Reproductive Medicine, Third Affiliated Hospital of Guangzhou Medical University, Guangzhou 510150, China. ${ }^{3}$ Department of Radiation and Medical Oncology, Zhongnan Hospital of Wuhan University, No.169 Donghu Road, Wuhan 430072, China. ${ }^{4}$ Gene Editing Center, School of Life Science and Technology, ShanghaiTech University, 100 Haike Rd., Pudong New Area, Shanghai 201210, China. ${ }^{5}$ Shanghai Institute for Advanced Immunochemical Studies, ShanghaiTech University, Shanghai 201210, China. ${ }^{6}$ Department of Laboratory Medicine, Zhongnan Hospital 
of Wuhan University, Wuhan University, No.169 Donghu Road, Wuhan 430072, China. ${ }^{7}$ Institute for Brain Research and Rehabilitation, Guangdong Key Laboratory of Mental Health and Cognitive Science, Center for Studies of Psychological Application, South China Normal University, Guangzhou 510631, China. ${ }^{8}$ State Key Laboratory of Respiratory Disease/National Clinical Research Center for Respiratory Disease/National Center for Respiratory Medicine/Guangzhou Institute of Respiratory Health/The First Affiliated Hospital of Guangzhou Medical University, Guangzhou 510120, China. ${ }^{9}$ School of Physical Science and Technology, ShanghaiTech University, 100 Haike Rd., Pudong New Area, Shanghai 201210, China. ${ }^{10}$ Shenzhen Branch, Guangdong Laboratory of Lingnan Modern Agriculture, Genome Analysis Laboratory of the Ministry of Agriculture and Rural Affairs, Agricultural Genomics Institute at Shenzhen, Chinese Academy of Agricultural Sciences, Shenzhen, China.

Received: 16 August 2021 Accepted: 5 November 2021

Published online: 02 December 2021

\section{References}

1. Berger MF, Mardis ER. The emerging clinical relevance of genomics in cancer medicine. Nat Rev Clin Oncol. 2018;15(6):353-65. https://doi.org/ 10.1038/s41571-018-0002-6

2. Li Y, Li S, Wang J, Liu G. CRISPR/Cas systems towards next-generation biosensing. Trends Biotechnol. 2019;37(7):730-43. https://doi.org/10.1016/j. tibtech.2018.12.005.

3. Teng F, Guo L, Cui T, Wang XG, Xu K, Gao Q, et al. CDetection: CRISPRCas 12b-based DNA detection with sub-attomolar sensitivity and singlebase specificity. Genome Biol. 2019;20(1):132. https://doi.org/10.1186/ s13059-019-1742-z.

4. Myhrvold C, Freije CA, Gootenberg JS, Abudayyeh OO, Metsky HC, Durbin $A F$, et al. Field-deployable viral diagnostics using CRISPR-Cas13. Science. 2018;360(6387):444-8. https://doi.org/10.1126/science.aas8836.

5. Wang $X$, Shang $X$, Huang $X$. Next-generation pathogen diagnosis with CRISPR/Cas-based detection methods. Emerg Microbes Infect. 2020;9(1):1682-91. https://doi.org/10.1080/22221751.2020.1793689.

6. Gootenberg JS, Abudayyeh OO, Lee JW, Essletzbichler P, Dy AJ, Joung $J$, et al. Nucleic acid detection with CRISPR-Cas13a/C2c2. Science. 2017;356(6336):438-42. https://doi.org/10.1126/science.aam9321.

7. Chen JS, Ma E, Harrington LB, Da Costa M, Tian X, Palefsky JM, et al. CRISPR-Cas 12 a target binding unleashes indiscriminate single-stranded DNase activity. Science. 2018;360(6387):436-9. https://doi.org/10.1126/ science.aar6245.

8. Ding X, Yin K, Li Z, Lalla RV, Ballesteros E, Sfeir MM, et al. Ultrasensitive and visual detection of SARS-CoV-2 using all-in-one dual CRISPRCas12a assay. Nat Commun. 2020;11(1):4711. https://doi.org/10.1038/ s41467-020-18575-6.

9. Ma P, Meng Q, Sun B, Zhao B, Dang L, Zhong M, et al. MeCas12a, a highly sensitive and specific system for COVID-19 detection. Adv Sci (Weinh). 2020;2001300. https://doi.org/10.1002/advs.202001300.

10. Lobato IM, O'Sullivan CK. Recombinase polymerase amplification: Basics, applications and recent advances. Trends Anal Chem. 2018;98:19-35. https://doi.org/10.1016/j.trac.2017.10.015.

11. Daver N, Cortes J, Ravandi F, Patel KP, Burger JA, Konopleva M, et al. Secondary mutations as mediators of resistance to targeted therapy in leukemia. Blood. 2015;125(21):3236-45. https://doi.org/10.1182/ blood-2014-10-605808.

12. Petiti J, Rosso V, Croce E, Franceschi V, Andreani G, Dragani M, et al. Highly sensitive detection of IDH2 mutations in acute myeloid leukemia. J Clin Med. 2020;9(1). https://doi.org/10.3390/jcm9010271.

13. Yang $\mathrm{H}$, Ye D, Guan $\mathrm{KL}$, Xiong $\mathrm{Y}$. IDH1 and IDH2 mutations in tumorigenesis: mechanistic insights and clinical perspectives. Clin Cancer Res. 2012;18(20):5562-71. https://doi.org/10.1158/1078-0432.CCR-12-1773.

14. Paez JG, Janne PA, Lee JC, Tracy S, Greulich H, Gabriel S, et al. EGFR mutations in lung cancer: correlation with clinical response to gefitinib therapy. Science. 2004;304(5676):1497-500. https://doi.org/10.1126/scien ce.1099314

15. Wang J, Kong G, Liu Y, Du J, Chang Yl, Tey SR, et al. Nras(G12D/+) promotes leukemogenesis by aberrantly regulating hematopoietic stem cell functions. Blood. 2013;121(26):5203-7. https://doi.org/10.1182/ blood-2012-12-475863.

\section{Publisher's Note}

Springer Nature remains neutral with regard to jurisdictional claims in published maps and institutional affiliations.
Ready to submit your research? Choose BMC and benefit from:

- fast, convenient online submission

- thorough peer review by experienced researchers in your field

- rapid publication on acceptance

- support for research data, including large and complex data types

- gold Open Access which fosters wider collaboration and increased citations

- maximum visibility for your research: over 100M website views per year

At BMC, research is always in progress.

Learn more biomedcentral.com/submissions 